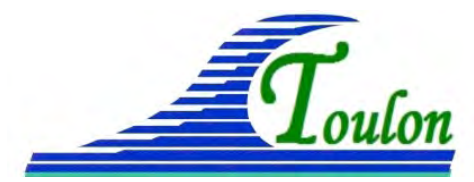

XIV $V^{\text {èmes }}$ Journées Nationales Génie Côtier - Génie Civil Toulon, 29 juin au $1^{\text {er juillet } 2016}$

DOI:10.5150/jngcgc.2016.001 @ Editions Paralia CFL disponible en ligne - http://www.paralia.fr - available online

\title{
Interactions entre la zone de déferlement et le jet de rive sur une plage à terrasse
}

\author{
Rafael ALMAR ${ }^{1}$, Pedro ALMEIDA ${ }^{1}$, Chris BLENKINSOPP ${ }^{2}$
}

1. LEGOS (CNRS-IRD-CNES- Université de Toulouse III), Toulouse, France. rafael.almar@ird.fr, luis.pedro.almeida@legos.obs-mip.fr

2. Dpt of Architecture and Civil Engineering, U. of Bath, UK. Blenkinsopp@bath.ac.uk

\section{Résumé :}

Le rôle de la dynamique couplée zone de déferlement-jet de rive sur la réflexion des vagues est étudiée grâce à des données collectées sur la plage à terrasse de Grand Popo au Bénin (Golfe de Guinée, Afrique de l'Ouest). Ces analyses sont effectuées à partir de spectres directionnels de vagues au large, des relevés topographiques et un suivi vidéo. Nos résultats révèlent que le jet de rive peut être une composante non négligeable du bilan énergétique littoral (14\% de la dissipation totale) et est étroitement liée à la réflexion. La réflexion dépend des caractéristiques des vagues à l'initiation du jet de rive (contrôlées par la saturation de la zone de déferlement), et la pente de la zone de jet de rive, qui varie avec la marée et l'évolution morphologique. Ces résultats montrent qu'il existe une fréquence de coupure du spectre réfléchi (i.e. filtre passe bas) liée à la saturation dans la zone de jet de rive dépendant de la pente de haut de plage. Un modèle à résolution de phase est utilisé pour étudier l'influence de la largeur de la terrasse, la pente du haut de plage et le niveau de marée sur la réflexion. Cette étude met en avant le rôle du système couplé zone de déferlement - jet de rive et souligne les interactions clés entre une évolution morphologique du haut de plage, rapide, et l'hydromorphodynamique de la zone de déferlement.

Mots-clés : Grand Popo, Benin (Golfe de Guinée), Jet de rive, Réflexion des vagues, Onde stationnaire.

\section{Introduction}

La réflexion des vagues à la côte peut atteindre 60 à $80 \%$ de l'énergie incidente et varie fortement selon les conditions hydro-morphologiques (ELGAR et al., 1994). Améliorer notre connaissance de cette réflexion est essentiel pour le bilan énergétique littoral et sa rétroaction sur les vagues incidentes (ARDHUIN \& ROLAND, 2012). De plus, le lien supposé entre réflexion et morphologie du haut de plage permet d'envisager une estimation distante de la morphologie du haut de plage par la mesure au large des vagues réfléchies (WATSON, 2012). Les premières études en canal ont montré un lien entre la réflexion et le ratio entre la cambrure de vagues et la pente de plage, c'est-à-dire entre l'accélération verticale et la gravité, qui peut se résumer par le paramètre d'Iribarren (IRIBARREN \& NOGALES, 1949 ; BATTJES, 1974). Des observations de 


\section{Thème 1 - Hydrodynamique côtière}

terrain ont montré que ces prédictions sont médiocres pour les vagues irrégulières et des profils bathymétriques complexes (GUZA \& THORNTON, 1982 ; ELGAR et al., 1994). Des études plus récentes, bien que portant principalement sur la conception de structures d'ingénierie (SUTHERLAND \& O’DONOGHUE, 1998, entre autres), ont souligné le rôle clé joué par la dynamique de la zone de jet de rive dans le contrôle de la phase et l'énergie des ondes réfléchies. Initialement développé par HUGHES et FOWLER (1995), la réflexion est liée au rapport de la longueur d'ondes du jet de rive et de son excursion horizontale.

La saturation de l'énergie des vagues dans la zone de déferlement est liée au ratio de la hauteur des vagues sur la profondeur et décrit l'intensité du déferlement des vagues (SALLENGER \& HOLMAN, 1985). Il a été largement observé que la bande incidente ( $\mathrm{T}<20 \mathrm{~s}$ ) est généralement saturée alors que la bande infragravitaire ( $\mathrm{T}>20 \mathrm{~s}$ ) sature rarement (RUESSINK et al., 1998), bien que des observations récentes ont montré que la saturation pourrait s'étendre aux ondes infragravitaires (Ruggiero \& HOLMAN, 2004, SENECHAL et al., 2011) qui peuvent même déferler (DE BAKKER et al., 2014). Cette saturation reflète les transformations de la zone de déferlement (GUEDES et al., 2013), mais également les processus de la zone de jet de rive tels que les appariements ou les interactions entre jet incident et descendant (BROCCHINI \& BALDOCK, 2008). Supposé crucial, le lien entre la dynamique de jet de rive et la réflexion n'a pas été clairement établi, en particulier le rôle de la saturation pour les spectres à large bande.

Dans cet article, nous étudions le lien entre la dynamique couplée zone de déferlementjet de rive avec la réflexion pour une plage à terrasse, Grand Popo, Bénin, dans le Golfe de Guinée, une des régions où les plus fortes réflexions ont été observées (ARDHUIN \& ROLAND, 2012 ; CRAWFORD et al., 2015). Nous montrons que la réflexion est principalement régie par la modulation du déferlement sur la terrasse, et le spectre réfléchi dépend essentiellement de la pente de la zone de jet de rive active.

\section{Données et méthodes}

Une campagne de mesures a été menée en Mars 2014 à Grand Popo, Bénin (Golfe de Guinée, Afrique de l'Ouest, figure 1), une côte sableuse ouverte exposées aux longues houles de l'Atlantique Sud (ALMAR et al., 2014a). La plage de Grand Popo est intermédiaire à réfléchissante $(\Omega>2)$, de type terrasse de bas de plage, micro-tidale et dominée par les vagues (moyenne annuelle, $H s=1.4 \mathrm{~m}, T p=9.4 \mathrm{~s}$, venant du SW, $\mathrm{RTR} 1$ ). Le sédiment est de taille moyenne avec $\mathrm{D} 50=0.6 \mathrm{~mm}$. Les vagues sont mesurées à $12 \mathrm{~m}$ de profondeur, la terrasse est instrumentée avec 2 lignes qui comprennent des capteurs de pression, un courantomètre haute fréquence, et deux sections de 20 piquets suivis par vidéo (chaque $\mathrm{m}$ ) pour la mesure à haute fréquence (25 $\mathrm{Hz}$ ) de la transformation des vagues à travers la zone de déferlement et de jet de rive. Un suivi topographique journalier est réalisé au GPS différentiel et un levé bathymétrique est obtenu par échosondeur. 


\section{XIV ${ }^{\text {èmes }}$ Journées Nationales Génie Côtier - Génie Civil \\ Toulon, 29 juin au $1^{\text {er }}$ juillet 2016}

Des simulations numériques sont réalisées en utilisant le modèle Boussinesq à résolution de phase non-linéaire d'ordre 4 SERR1D (CIENFUEGOS et al., 2010). Ce modèle a déjà été validé avec des données de laboratoire pour des conditions très nonlinéaires sur une plage en pente douce (MICHALLET et al., 2014). Le modèle a montré de bonnes performances en représentant à la fois la dynamique des ondes courtes et le transfert énergétique vers l’infragravitaire.
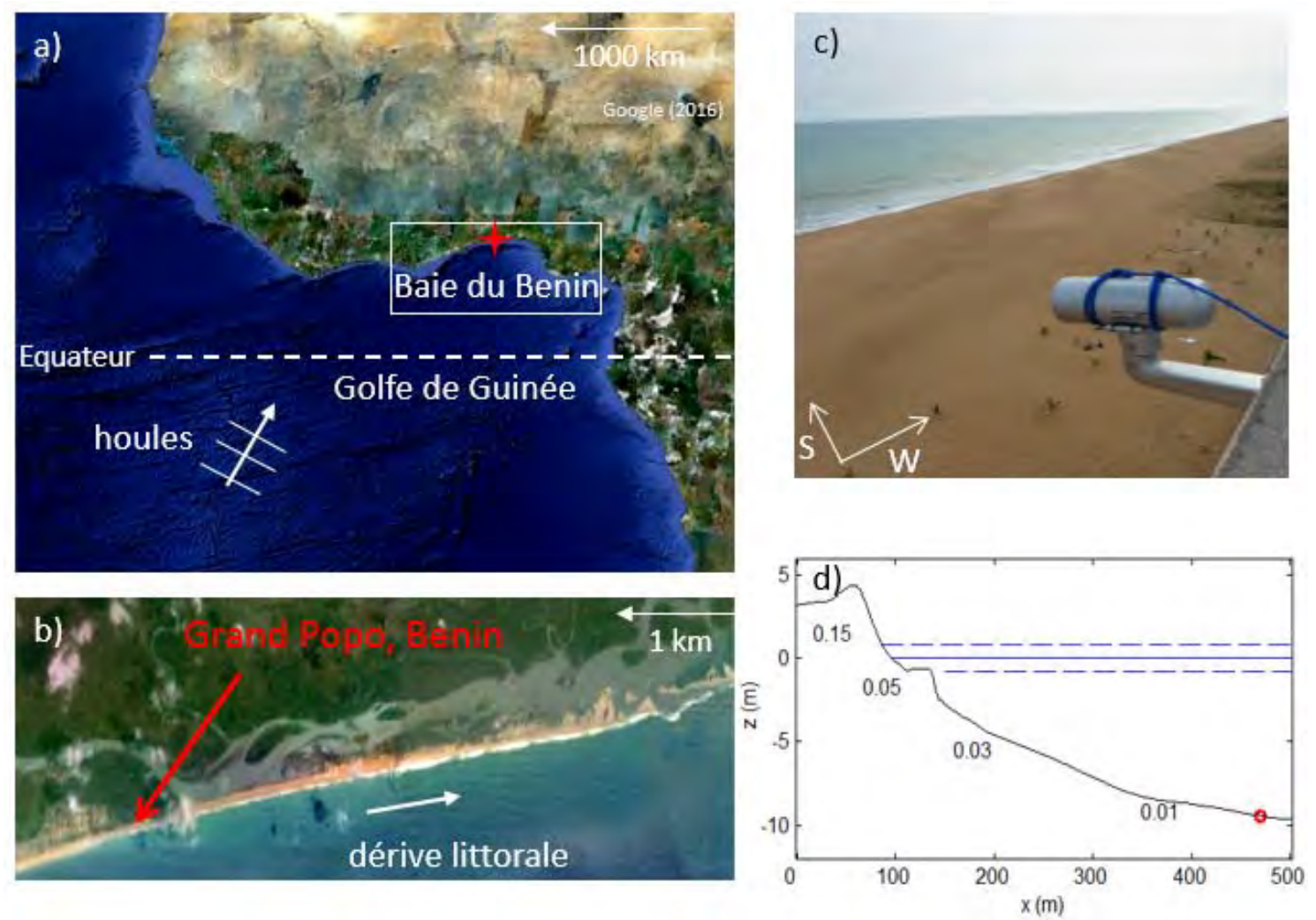

Figure 1. Site d'étude, Grand Popo, Bénin. a) et b) localisation, c) système vidéo et d) profil bathymétrique. Le cercle rouge montre l'emplacement de l'ADCP.

\section{Résultats et discussion}

La figure 2 montre que la hauteur des vagues a diminué durant la campagne, avec une augmentation du marnage. Alors que la plage s'est raidie, la pente de la zone de jet de rive active, fortement modulée par la marée a légèrement diminuée. Ce contrôle croissant par la marée sur les conditions hydrodynamiques se retrouve dans le paramètre du ratio de la hauteur des vagues sur la profondeur.

La réflexion est-elle induite par la dynamique de la zone de déferlement ou de jet de rive ? La réflexion atteint $8 \%$ en moyenne, variant de 4 à $12 \%$. La dissipation induite par le déferlement, l'énergie du jet de rive et la réflexion varient avec les conditions de vagues, la marée et l'évolution morphologique. Une régression linéaire multiple montre que le déferlement et le jet de rive dissipent 71 et 14\% respectivement de l'énergie 
incidente, et varient sensiblement (figure 2.e). L'énergie du jet de rive peut même être prédominante à certains moments, par exemple à marée haute lorsque le déferlement sur la terrasse est faible.

Le jet de rive, un filtre passe bas ? Les spectres d'énergie de jet de rive sont calculés à partir de 20 min de séquences vidéo. Avec l'idée de lier la saturation du jet de rive à la réflexion, et en suivant la méthode décrite dans RUESSINK et al. (1998), la plus basse fréquence saturée est extraite à partir des spectres (figure 3) comme la fréquence de coupure $f c_{j e t}$ de rive. La plus haute fréquence réfléchie $f c_{\text {offshore }}$ est également calculée à partir des spectres de direction des vagues au large. Un ajustement raisonnable $\left(\mathrm{r}^{2}=\right.$ 0.42 , significatif à $95 \%$ ) est obtenu entre $f c_{\text {jet de rive }}$ et $f c_{\text {offshore }}$ avec seulement un biais mineur, $f c_{\text {offshore }}=0.89 f c_{\text {jet de rive. }}$ La corrélation de $f c_{\text {offshore }}$ est plus grande avec $\alpha(0.51)$ qu'avec $f_{\text {offshore }}(0.32)$, indiquant que cette fréquence de coupure dépend de $\alpha$ et non des vagues.

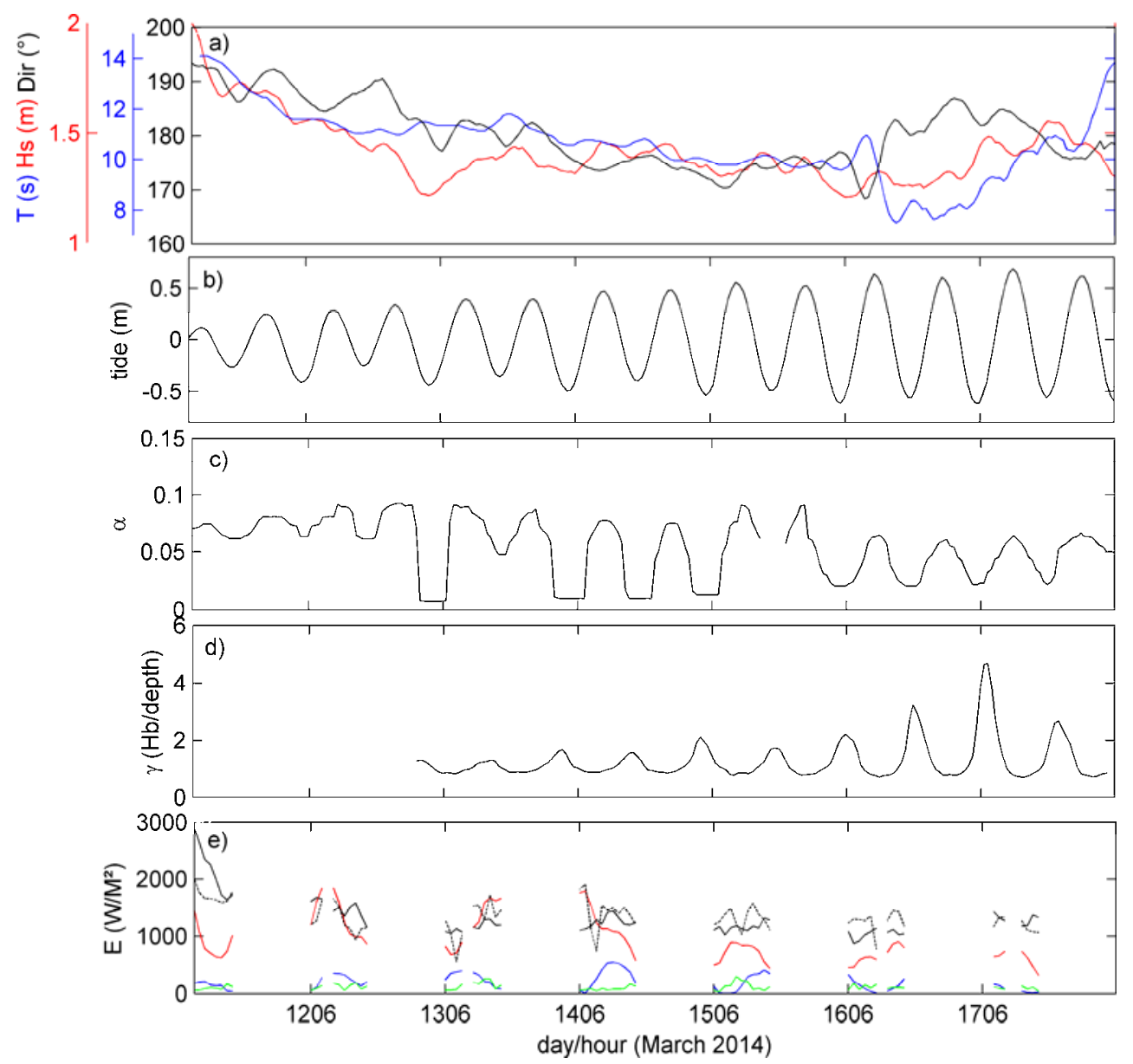

Figure 2. Séries temporelle a) de vagues, b) marée, c) pente de jet de rive actif, d) ratio hauteur de vagues sur profondeur sur la terrasse et e) énergie incidente (noir continu), dissipée au déferlement (rouge, selon HALLER \& CATALAN, 2009), jet de rive (bleu, selon ALMAR et al., 2014b), réfléchie (vert) et reconstruite (noir discontinu). 


\section{XIV ${ }^{\text {èmes }}$ Journées Nationales Génie Côtier - Génie Civil \\ Toulon, 29 juin au $1^{\text {er }}$ juillet 2016}
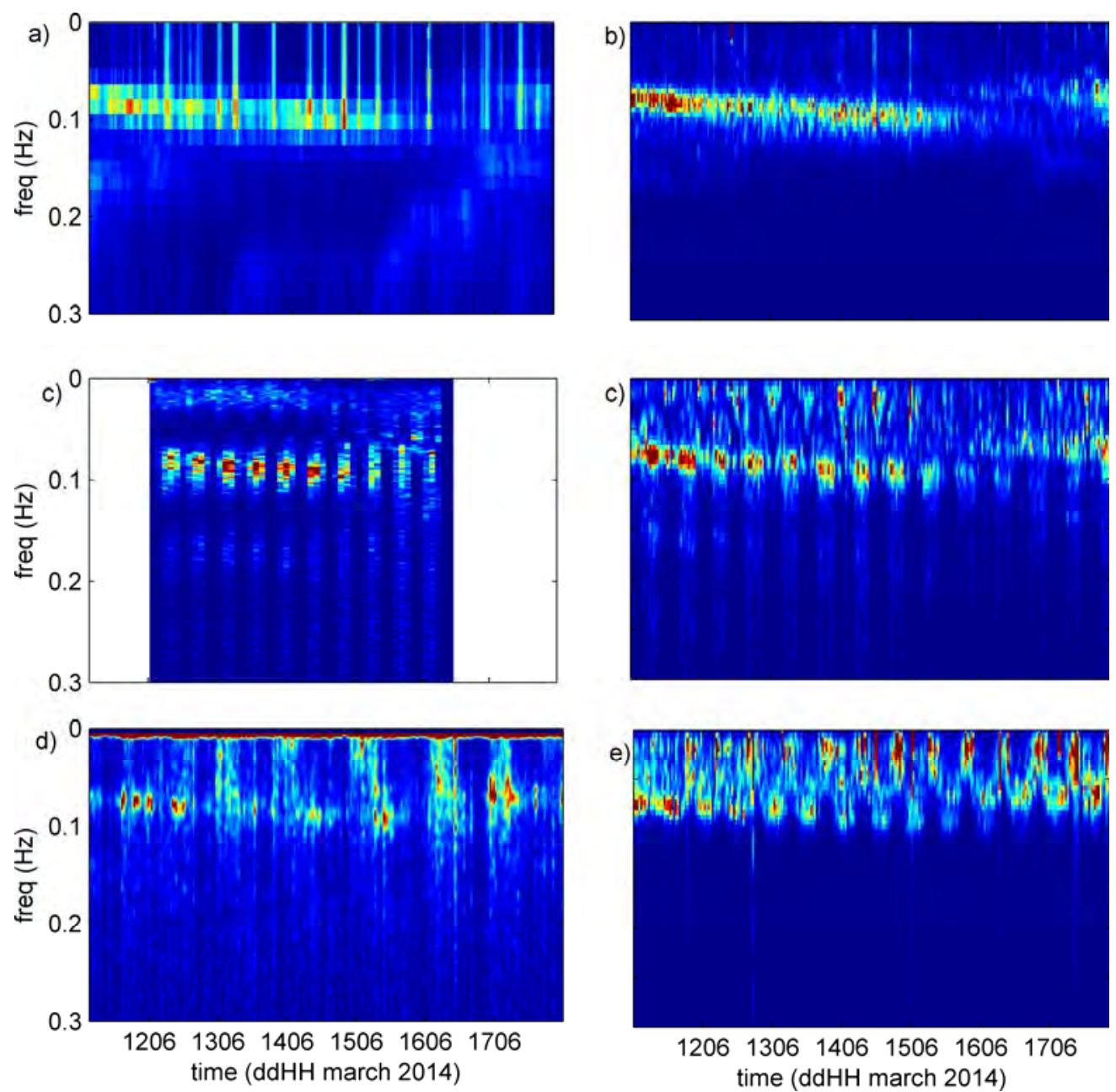

Figure 3. Spectres de vagues, observés à gauche à l'ADCP (haut), sur la terrasse (milieu) et dans le jet de rive (bas) et modélisés à droite.

Sensibilité de la réflexion à la marée, la largeur de terrasse et la pente de jet de rive. 20 min de séries d'élévation de surface au niveau de l'ADCP sont propagées sur le profil bathymétrique moyen (figure 1). La figure 3 montre que le modèle reproduit les transferts de fréquences qui incluent la génération d'ondes secondaires à marée haute et infragravitaires à marée basse. La figure 4 montre qu'un élargissement de la terrasse déplace le système vers des conditions plus dissipatives, ce qui augmente le transfert d'énergie vers la bande infragravitaire. L'effet de la marée est similaire avec une saturation de la bande incidente lorsque le niveau baisse. La réflexion augmente avec la pente du haut de plage, la fréquence de coupure augmentant également. Le niveau de marée sur la terrasse n'a que peu d'influence sur le réfléchi. A noter une faible diminution lorsque le déferlement est faible et que le spectre incident en début de jet de rive ne s’est pas déplacé vers les basses fréquences et n’a moins de capacité de réflexion. 

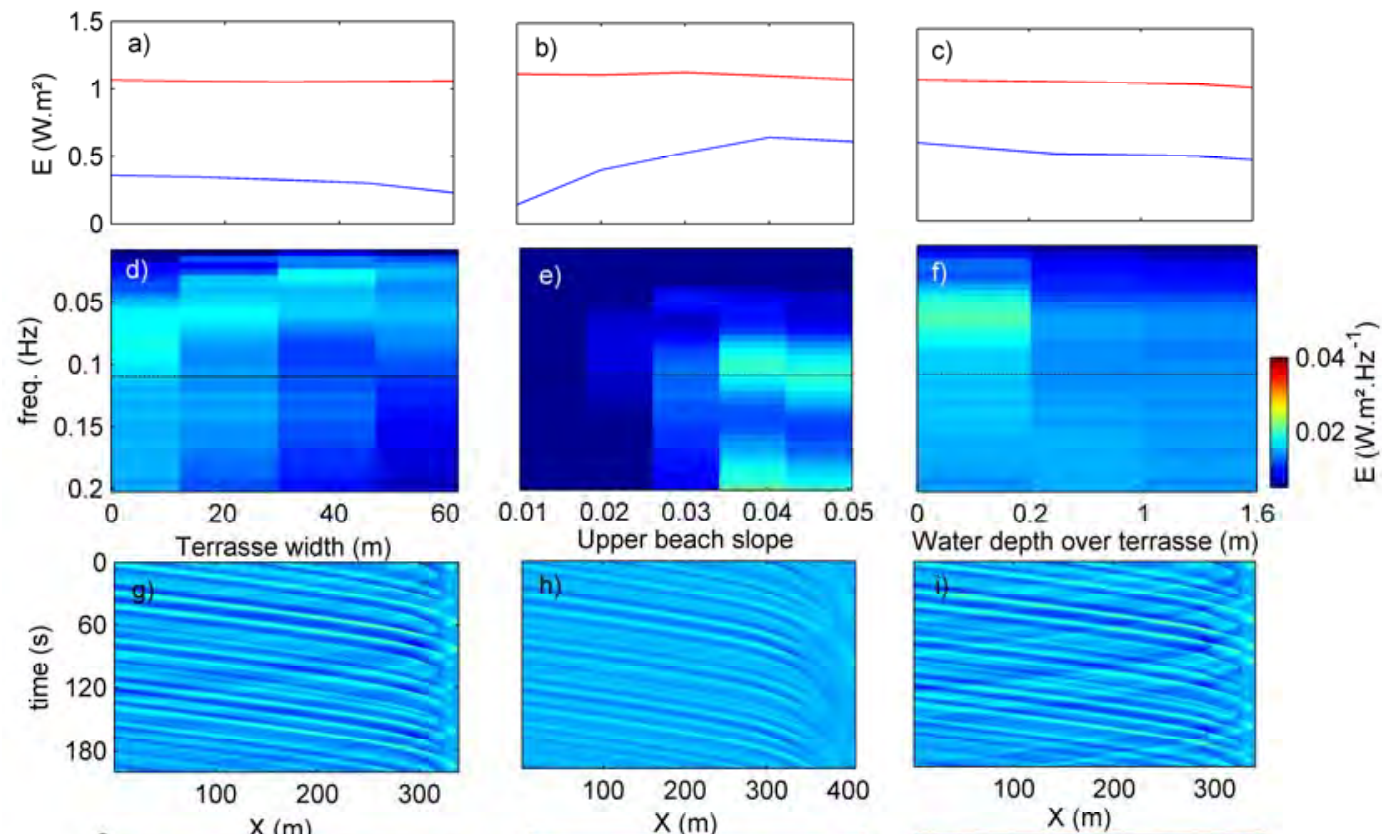

Water depth over terrasse $(\mathrm{m})$
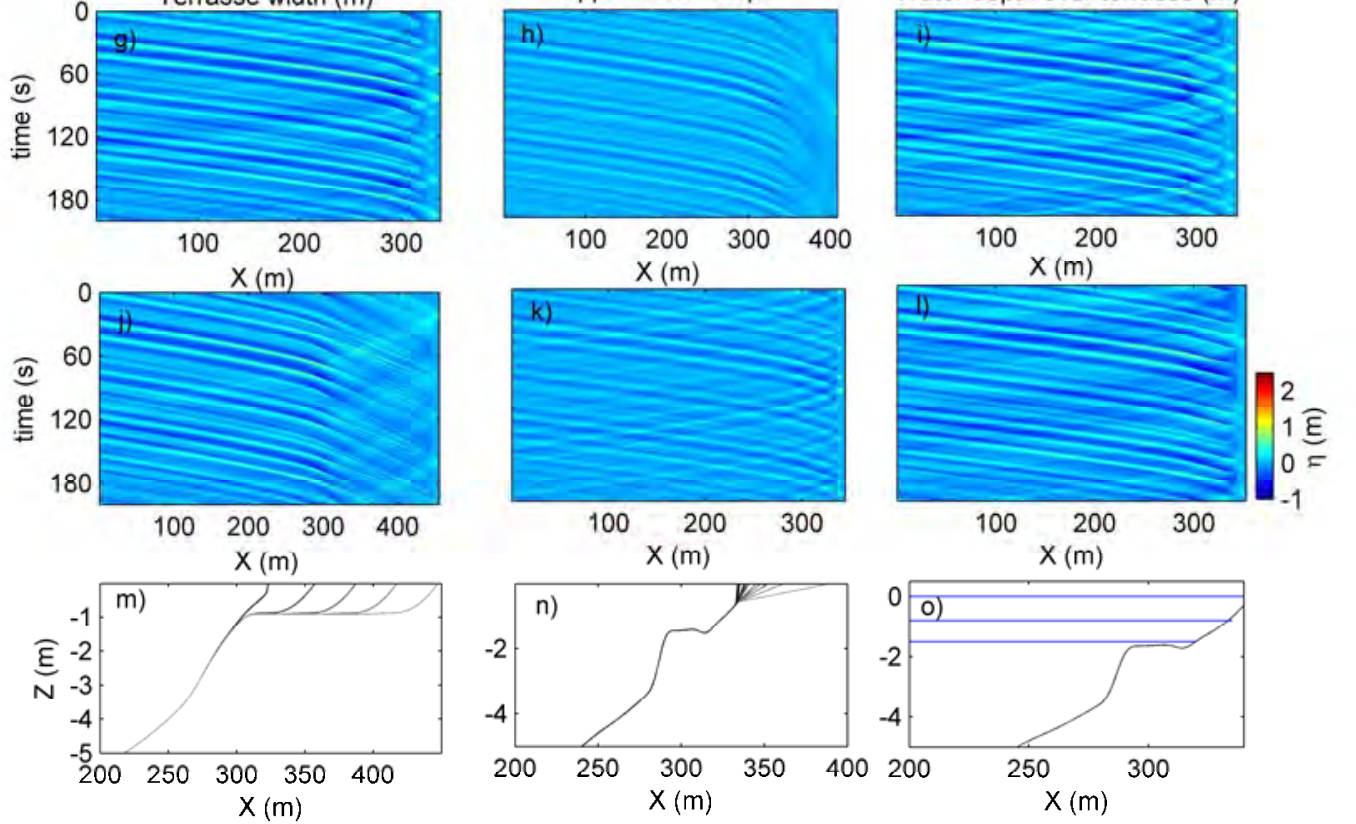

Figure 4. Sensibilité de la réflexion à la largeur de la terrasse (gauche), pente de haut de plage (milieu) et hauteur d'eau (marée) sur la terrasse (droite). a-c) énergie incidente (bleu) et réfléchie (rouge), d-f) spectres réfléchis, g-i) et j-l) sont des Hovmoller d'élévation de surface pour les valeurs minimales et maximales des paramètres, respectivement. m-o) montre les bathymétries et niveaux d'eau utilisés.

\section{Conclusions}

Cet article montre l'influence du système zone de déferlement-jet de rive sur la réflexion pour la plage à terrasse de Grand Popo, Bénin. Nos observations, combinant l'imagerie vidéo et la mesure des vagues en eau profonde, montrent le rôle clé joué par le jet de rive dans la réflexion. Le jet de rive contribue en moyenne à $14 \%$ de la dissipation de l'énergie incidente et augmente à marée haute, lorsque le déferlement est limité. Un bon accord est obtenu entre les fréquences de coupure calculées à partir du jet de rive et des spectres au large. Contrairement à la réflexion, cette fréquence de coupure dépend principalement de la pente du haut de plage. 


\section{XIV ${ }^{\text {èmes }}$ Journées Nationales Génie Côtier - Génie Civil \\ Toulon, 29 juin au $1^{\text {er }}$ juillet 2016}

Le modèle Boussinesq Serr1D montre de bonnes performances pour décrire les transferts en fréquence sur la terrasse. Le modèle est utilisé pour étudier l'influence de la largeur de la terrasse, de la pente de haut de plage et de la marée sur les vagues réfléchies. L'influence la plus frappante est jouée par la pente de haut de plage qui contrôle étroitement les vagues réfléchies. Pour des fortes pentes de haut de plage, le contrôle du réfléchi sur l'incident augmente, représentant une rétroaction du jet de rive sur le spectre incident dans la zone de déferlement qui tend vers celui réfléchi (contrôlé par la pente du haut de plage), favorisant la condition d'apparition d'une onde stationnaire ou même résonnante.

\section{Remerciements}

Les auteurs remercient les participants à la campagne de Grand Popo 2014, en particulier les collègues béninois et les forces navales qui ont permis que se réalise cette campagne. Le projet COASTVAR (ANR-14-ASTR-0019) a également reçu un financement INSU LEFE et EC2CO (2013-2014).

\section{Références bibliographiques}

ARDHUIN F., ROLAND A. (2012). Coastal wave reflection, directional spread, and seismoacoustic noise sources. J. of Geophysical Research, Vol.117, C00J20. http://dx.doi.org/10.1029/2011jc007832

ALMAR R., DU PENHOAT Y., HONKONNOU N., CASTELLE B., LAIBI R., ANTHONY E., SENECHAL N., DEGBE G., CHUCHLA R., SOHOU Z., DOREL M. (2014a). The Grand Popo experiment, Benin. Journal of Coastal Research, SI 70, pp 651-656. http://dx.doi.org/10.2112//170-110.1

ALMAR R., MICHALLET H., CIENFUEGOS R., BONNETON P., RUESSINK B.G., TISSIER M. (2014b). On the use of the Radon Transform in studying nearshore wave dynamics. Coastal Engineering, Vol. 92, pp 24-30. http://dx.doi.org/10.1016/j.coastaleng.2014.06..008

BATTJES J. (1974). Surf similarity. 14th Coastal Engineering Conference Am. Soc. of Civ. Eng., Copenhagen, Denmark, pp 466-480. http://dx.doi.org/10.1061/9780872621138.029 BROCCHINI M., BALDOCK T. (2008). Recent advances in modeling jet de rive zone dynamics: Influences of surf-jet de rive interaction on nearshore hydrodynamics and morphodynamics. Reviews of Geophysics, Vol. 46(3), RG3003. http://dx.doi.org/10.1029/2006RG000215

CIENFUEGOS R., BARTHELEMY E., BONNETON P. (2010). A wave-breaking model 34 for Boussinesq-type equations including roller effects in the mass conservation equation. J. Waterw. Port Coastal Oc. Eng., Vol. 136, pp 10-26.

CRAWFORD W., BALLU V., BERTIN X., KARPYTCHEV M. (2015). The sources of deep ocean infragravity waves observed in the North Atlantic Ocean. Journal of Geophysical Research: Oceans, Vol. 120(7). http://dx.doi.org/10.1002/2014JC010657 
DE BAKKER A., TISSIER M., RUESSINK B. (2014). Shoreline dissipation of infragravity waves. Continental Shelf Research, Vol. 72(1), pp 73-82. http://dx.doi.org/10.1016/j.csr.2013.11.013

ELGAR S., HERBERS T., GUZA R. (1994). Reflection of ocean surface gravity waves from a natural beach. J. Physical. Oceanogr., Vol. 24(7), pp 1503-1511. http://dx.doi.org/10.1175/1520-0485(1994)024<1503:roosgw>2.0.co;2

GUEDES R., BRYAN K., COCO G. (2013). Observations of wave energy fluxes and jet de rive motions on a low-sloping, dissipative beach. Journal of Geophysical Research, Vol. 118(7), pp 1-19. http://dx.doi.org/10.1002/jgrc.20267

GUZA R., THORNTON E. (1982). Jet de rive oscillations on a natural beach. J. Geophys. Res., Vol. 87, pp 483-491. http://dx.doi.org/10.1029/JC087iC01p00483

HALLER M.C., CATALAN P. (2009). Remote sensing of wave roller lengths in the laboratory. J. Geophys. Res., Vol. 114, C07022. http://dx.doi.org/10.1029/2008JC005185

HUGHES S., FOWLER J. (1995). Estimating wave-induced kinematics at sloping structures. Journal of Waterway, Port, Coastal, and Ocean Engineering, Vol. 121(4), pp 209-215. http://dx.doi.org/10.1061/(ASCE)0733-950X(1995)121:4(209)

IRIBARREN C.R., NOGALES C. (1949). Protection des ports, Proceedings XVIIth International Navigation Congress, Section II, Communication 4, Lisbon, pp. 31-80.

MICHALLET H., RUESSINK G., ROCHA M.V.L., DE BAKKER A., VAN DER A.D., RUJU A., SILVA P.A., SENECHAL N., MARIEU V., TISSIER M., ALMAR R., ABREU T., BIRRIEN F., VIGNAL L., BARTHELEMY E., MOUAZE D., CIENFUEGOS R., WELLENS P. (2014). GLOBEX: Wave dynamics on a shallow sloping beach. Proceedings of the HYDRALAB IV Joint User Meeting, Lisbon, July. RUESSINK B., KLEINHANS M., VAN DEN BEUKEL P. (1998). Observations of jet de rive under highly dissipative conditions. J. Geophys. Res., Vol. 103, pp 3111-3118. http://dx.doi.org/10.1029/97JC02791

RUGGIERO P., HOLMAN R. (2004). Wave run-up on a high-energy dissipative beach. J. Geophys. Res., C06025. http://dx.doi.org/10.1029/2003jc002160

RUJU A., LARA J.L., MICHALLET H., SÉNÉCHAL N., LOSADA I.J. (2013). Transient jet de rive motions on a gently sloping beach. Proceedings Coastal Dynamics. SALLENGER A., HOLMAN R. (1985). Wave energy saturation on a natural beach of variable slope. J. Geophys. Res. Vol. 90-C6/11, pp 939-944. http://dx.doi.org/10.1029/jc090ic06p11939

SENECHAL N., COCO G., BRYAN K., HOLMAN R. (2011). Wave runup during 355 extreme storm conditions. Journal of Geophysical Research, Oceans, Vol. 116, C07032. http://dx.doi.org/10.1029/2010JC006819

SUTHERLAND J., O'DONOGHUE T. (1998). Characteristics of wave reflection spectra. J. Waterw. Port Coastal Ocean Eng., Vol. 124(6), pp 303-311. http://dx.doi.org/10.1061/(ASCE)0733-950X(1998)124:6(303)

WATSON, D.H. (2012). Wave reflection on a two-slope beach. Thesis report of the Naval Postgraduate school, California, USA (Advisors. Herbert, T.; MacMahan, J.) 